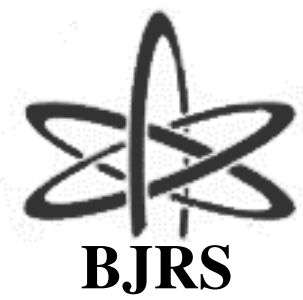
BRAZILIAN JOURNAL
$\mathrm{OF}$
RADIATION SCIENCES
03-1A (2015) 01-12

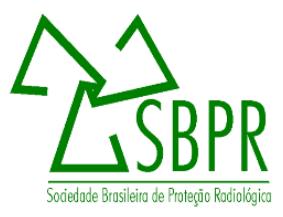

\title{
Obtenção de volumes tumorais em pilhas de imagens pet usando técnicas de segmentação de imagens coloridas
}

\author{
J. W. Vieira ${ }^{1,4}$, I. F. Vieira ${ }^{2}$, L. P. Cordeiro ${ }^{2}$, F. J. L. Filho ${ }^{1,4}$ e F. R. A. Lima ${ }^{2,3,4}$ \\ ${ }^{1}$ Instituto Federal de Educação, Ciência e Tecnologia de Pernambuco (IFPE Campus Recife) \\ 50740-540, Recife, PE, Brasil \\ jose.wilson@recife.ifpe.edu.br
}

2 Departamento de Energia Nuclear (DEN), Universidade Federal de Pernambuco (UFPE)

50740-54,0 Recife, PE, Brasil

igoradiologia@gmail.com, leoxofisico@gmail.com

3 Centro Regional de Ciências Nucleares do Nordeste (CRCN-NE), Comissão Nacional de Energia Nuclear

(CNEN), 50740-540, Recife, PE, Brasil

falima@cnen.gov.br

4 Escola Politécnica de Pernambuco(EPP), Universidade de Pernambuco (UPE)

50750-470 Recife, PE, Brasil

ferdinand.lopes@oi.com.br

\begin{abstract}
This work is demonstrated the step by step how to segment color images on the thoracic region of an adult in order to separate the tumor volume at present same conditions without significantly changing the values of R components (Red), G (Green) and B (Blue) color of the pixels . For information allowing build color map you need to segment and classify at appropriate intervals the colors present in the images. The used segmentation technique is to select a small rectangle with color samples of a given region and then erase with a specific color called "rubber" the other regions of the image. The tumor region was segmented into one of the images available and the procedure is shown in the tutorial format. All necessary computational tools have been implemented in DIP (Digital Image Processing), software developed by the authors. The results obtained besides permit the construction of the color map of the distribution of activity in PET images concentration will also be useful in future studies to insert tumors in voxel
\end{abstract}


phantoms for the purpose of carrying out dosimetry evaluations.

Keywords: segmetation, PET images, image processing

Palavras-chave: segmentação, imagens PET, processamento de imagens

\section{INTRODUÇÃO}

O objetivo deste trabalho é demonstrar os passos necessários para segmentar imagens PET (Positron Emission Tomography) visando separar um volume tumoral presente nas mesmas sem alterar significativamente os valores das componentes R (Red), G (Green) e B (Blue) das cores dos pixels. As imagens de pacientes submetidos a PET usualmente podem ser exibidas para diagnóstico como um mapa de cores onde cada cor é relacionada a uma concentração de atividade $(\mathrm{Bq} / \mathrm{ml})$. Para obter as informações qualitativas e quantitativas que permitam construir tais mapas é preciso segmentar e classificar em intervalos apropriados as cores presentes nas imagens.

Neste trabalho é apresentado um método para obter uma região de interesse (ROI) em uma imagem usando processamentos sequenciados que preservam as informações relacionadas com as cores originais. Para apresentação do método foram obtidas 20 imagens PNG (Portable Network Graphics) com 470 colunas e 256 linhas. Inicialmente, foi investigado se as imagens continham algum tipo de ruído oriundo da aquisição e/ou digitalização original, analisando perfis de linha das componentes RGB e também HSI (Hue, Saturation, Intensity) de uma imagem típica.

Se as imagens tiverem algum artefato indesejado, o passo seguinte demonstrado consistiu em aplicar um filtro de médias sobre as componentes RGB e também sobre a componente HSI de intensidade (I) de uma imagem típica. O melhor resultado pode ser avaliado usando imagens das diferenças entre as duas saídas deste passo. Também se pode aplicar sobre a imagem resultante uma filtragem com a imagem do laplaciano das componentes RGB e também da I para aumentar 
a nitidez. Por fim a segmentação da região tumoral pode ser realizada deixando a imagem com um fundo neutro e as cores que, então, serão associadas a valores de concentração de atividade.

A técnica de segmentação seleciona um pequeno retângulo com amostras de cores de uma dada região e, então, apaga com uma cor específica denominada "borracha" as demais regiões da pilha. A região tumoral foi segmentada na imagem típica e todas as ferramentas computacionais necessárias foram implementadas no DIP (Digital Image Processing), software desenvolvido pelos autores [VIEIRA, 2009].

Os resultados obtidos, além de permitirem a construção do mapa colorido da distribuição da concentração de atividade também serão úteis em futuros trabalhos para inserir tumores em fantomas de voxels e realizar avaliações dosimétricas.

\section{MATERIAIS E MÉTODOS}

Este trabalho foi desenvolvido no Laboratório de Dosimetria Numérica do IFPE Campus Recife, em computadores que têm como principais itens de configuração um processador Intel® Core ${ }^{\mathrm{TM}}$ i7 CPU X990 @ 3,47GHz, 24 GB de RAM e o sistema operacional Windows 7 Ultimate de 64 bits. Nestes computadores estão instalados os softwares aqui utilizados: o Microsoft Visual Studio 2013 Ultimate para 32 bits, o software de domínio público ImageJ 1.49b (http://imagej.nih.gov/ij/) e o DIP. As 20 imagens PET da região torácica de um adulto foram gentilmente cedidas pelo setor de radioterapia do Hospital Português de Recife.

A Figura 1 mostra, na janela principal do DIP, o menu onde foram concentradas as ferramentas de processamento das imagens disponíveis. Na sequência são apresentados os tópicos de processamento de imagens digitais no domínio espacial utilizados para melhorar a distribuição de pixels em imagens coloridas e, posteriormente, segmentar uma ROI nelas contida. Todas as implementações realizadas foram fundamentadas no livro de Gonzalez e Woods [GONZALES; WOODS, 2002]. 
Figura 1: Janela principal do software DIP, mostrando os itens do menu para processamento de imagens coloridas

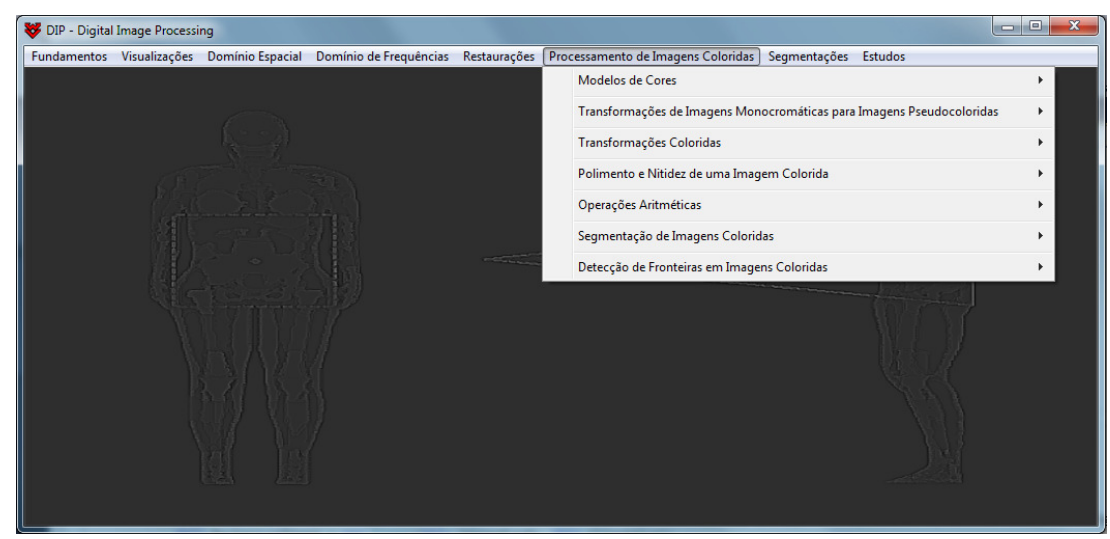

Fonte: Autores

Para suprimir possíveis artefatos indesejados, melhorar a distribuição de pixels e destacar ROIs em imagens coloridas, sem perdas substanciais das informações ali contidas, é preciso transformar cada pixel com base nas características dos pixels na sua vizinhança e em uma máscara cujos elementos são coeficientes que ponderam a importância de cada pixel da vizinhança adotada. As transformações que podem ser úteis às imagens utilizadas neste trabalho devem produzir saídas mais polidas e mais nítidas.

O polimento de uma imagem monocromática pode ser visto como uma operação de filtragem espacial na qual os coeficientes na máscara são iguais a 1. À medida que a máscara desliza sobre a imagem a ser polida, cada pixel é trocado pela média aritmética dos pixels sob ela.

Este conceito pode ser estendido para o polimento de imagens coloridas RGB, sendo que, neste caso, os tons de cinza das imagens monocromáticas são trocados pelos vetores das componentes,

$$
\mathbf{c}(x, y)=[R(x, y) \quad G(x, y) \quad B(x, y)] .
$$

Se $S_{x y}$ for o conjunto das coordenadas que define uma vizinhança de $K$ pixels, centrada em $(x, y)$, em uma imagem colorida RGB, o vetor das médias das componentes RGB nesta vizinhança é

$$
\overline{\mathbf{c}}(x, y)=\frac{1}{K}\left[\sum_{(s, t) \in S_{x y}} R(s, t) \sum_{(s, t) \in S_{x y}} G(s, t) \quad \sum_{(s, t) \in S_{x y}} B(s, t)\right] .
$$


As imagens das componentes deste vetor de médias podem ser construídas e polidas separadamente usando o processamento de vizinhança convencional para imagens monocromáticas. Após o polimento, as imagens resultantes, isto é, as componentes R, G e B transformadas, são agrupadas para constituírem a imagem polida. Cada pixel da imagem polida é produzido pelos valores dos tons de cinza nos pixels correspondentes das imagens $\mathrm{R}, \mathrm{G}$ e $\mathrm{B}$.

Para exemplificar o uso desta abordagem RGB, foi utilizado o arquivo Imagem_10.png contendo uma das imagens da pilha disponível. A Fig. 2 mostra a imagem original e suas componentes R, $\mathrm{G}$ e $\mathrm{B}$ (os rótulos $a, b, \ldots$, etc., que aparecem nesta e em outras figuras do trabalho seguem a orientação usual: da esquerda para a direita e de cima para baixo).

Figura 2: (a) Imagem RGB. (b) $R$, (c) $G$ e (d) $B$.
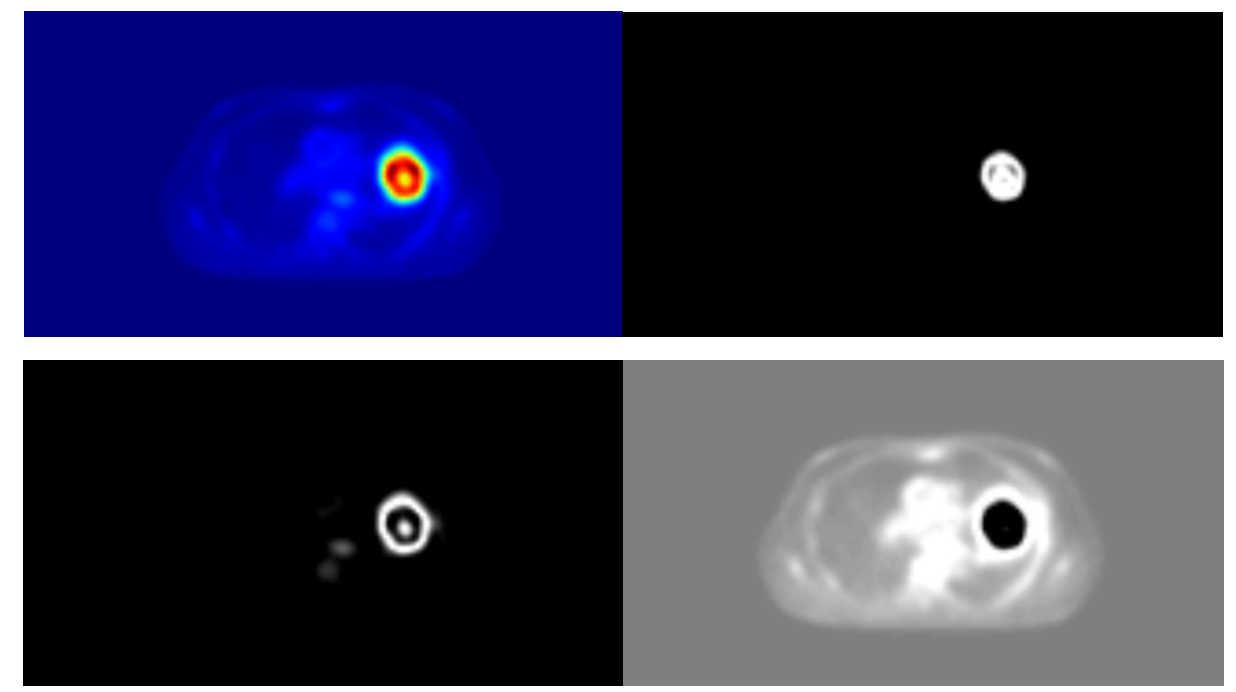

Fonte: Autores

Alternativamente, uma imagem colorida pode ser transformada usando a abordagem HSI que consiste em polir apenas a componente de intensidade deste modelo de cores. As Figuras 3a, b e c mostram as componentes HSI da Figura 2a. A Figura 3d é um esquema utilizado para definir as componentes HSI de uma imagem que foi implementado no software DIP. Note que, neste esquema, a cor vermelha corresponde a $\mathrm{H}=0^{\circ}$ e a magenta a $\mathrm{H}=360^{\circ}$.

$\mathrm{Na}$ Figura 4, a primeira imagem é o resultado do polimento da Figura 2a com uma máscara de média 3 x 3, usando a abordagem RGB; a $2^{\text {a }}$ imagem é o resultado da abordagem HSI. 
Teoricamente, uma vantagem importante de usar o modelo de cores HSI é que ele desacopla as informações de intensidade e cor. Isto o torna adequado para muitas técnicas de processamento de imagem e sugere que ele pode ser mais eficiente, realizando polimento apenas da componente I. Para ilustrar os méritos e/ou consequências desta abordagem, foi feito o polimento a imagem de intensidade da Figura 3c, sem alterar as componentes H e S. O resultado mostrado na segunda imagem da Figura 4 visualmente é diferente do resultado da abordagem RGB. As imagens das diferenças entre os dois polimentos, mostradas à direita em cada janela da Figura 4, confirmam esta expectativa. Isto se deve ao fato de que a média de dois pixels de cores diferentes é uma mistura das duas cores e não uma das cores originais. Quando há polimento apenas da imagem I, os pixels na segunda imagem na Figura 4 mantêm suas matização e saturação originais - e, consequentemente suas cores originais.

Figura 3: Imagens das componentes (a) H, (b) S e (c) I da Fig. 2a. (d) Esquema para definição das componentes HSI de uma imagem

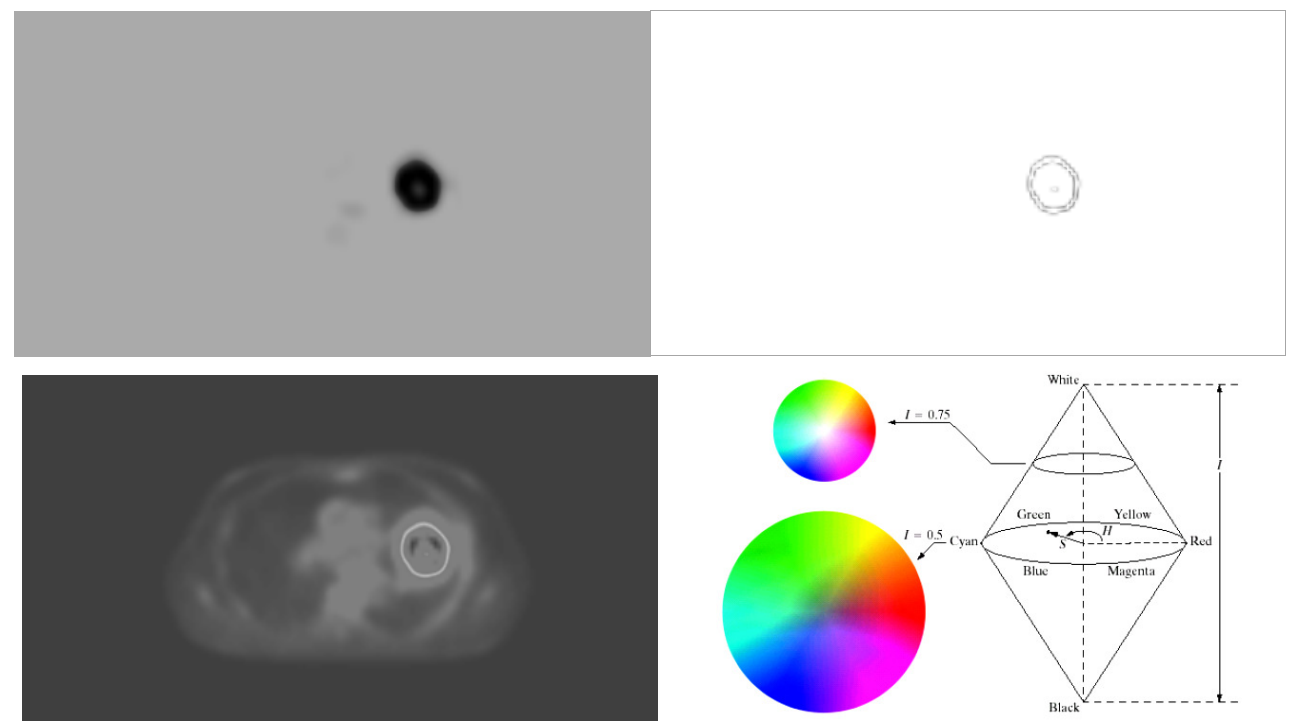

Fonte: Autores 
Figura 4: Polimento da Fig. 2 a com máscara de média $3 \times 3$, usando a abordagem RGB ( $1^{a}$ imagem) e a HSI ( $2^{a}$ imagem $)$.

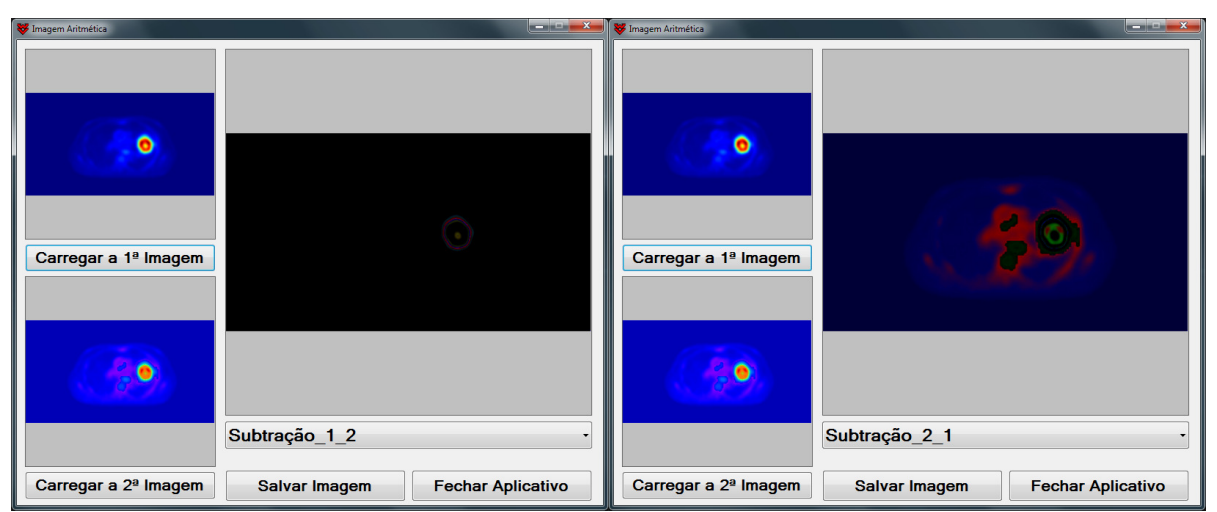

Fonte: Autores

A técnica aqui apresentada para melhorar a nitidez de imagens coloridas é baseada no laplaciano da função espacial $f(x, y)$ associada à intensidade de um pixel em uma imagem monocromática. Como o laplaciano é um operador de derivadas, seu uso destaca descontinuidades de tons de cinza em uma imagem e regiões que são separadas por bruscas variações nos tons. No modelo de cores RGB, o laplaciano do vetor c da equação (1) é

$$
\nabla^{2} \mathbf{c}(x, y)=\left[\begin{array}{lll}
\nabla^{2} R(x, y) & \nabla^{2} G(x, y) \quad \nabla^{2} B(x, y)
\end{array}\right]
$$

Como na equação (2), a imagem do laplaciano de uma imagem RGB pode ser obtida, calculando-se o laplaciano de cada uma das imagens componentes. Se $f(x, y)$ representa uma componente RGB, o laplaciano de $f(x, y)$ pode ser definido como

$$
\nabla^{2} f=\frac{\partial^{2} f}{\partial x^{2}}+\frac{\partial^{2} f}{\partial y^{2}}
$$

Como as derivadas de qualquer ordem são operações lineares, o laplaciano é um operador linear. A equação (4) deve ser expressa na forma discreta para ser útil em processamento de imagens. Há diversos modos de definir um laplaciano digital usando as vizinhanças. Porém, qualquer que seja a definição, ela tem que satisfazer as propriedades de uma derivada de segunda ordem, que 
são: uma derivada de segunda ordem (i) deve ser zero em áreas monótonas; (ii) deve ser não nula no início e no fim de degrau ou rampa de tons de cinza; e (iii) deve ser zero ao longo das rampas de inclinação constante. Uma das definições de derivada de segunda ordem digital mais populares é dada por:

$$
\left\{\begin{array}{l}
\frac{\partial^{2} f}{\partial x^{2}}=f(x+1, y)+f(x-1, y)-2 f(x, y) \\
\frac{\partial^{2} f}{\partial y^{2}}=f(x, y+1)+f(x, y-1)-2 f(x, y)
\end{array} .\right.
$$

A implementação do laplaciano digital (4), usando (5), pode ser realizada com o auxílio da máscara mostrada na Fig. 5a, que produz um resultado isotrópico para rotações com incrementos de $90^{\circ}$. As direções diagonais podem ser incorporadas na definição do laplaciano digital, adicionando-se mais termos às derivadas em (5). A máscara usada para implementar esta nova definição é mostrada na Figura 5b. Esta máscara produz resultados isotrópicos para incrementos de $45^{\circ}$.

Figura 5: (a) Máscara usada para implementar o laplaciano digital da equação (4), usando as derivadas definidas em (5). (b) Extensão da máscara (a) para incluir os vizinhos diagonais.

\begin{tabular}{|c|c|c||c|c|c|}
\hline 0 & 1 & 0 \\
\hline 1 & -4 & 1 & 1 & 1 \\
\hline 0 & 1 & 0 & -8 & 1 \\
\hline 1 & 1 & 1 \\
\hline
\end{tabular}

Fonte: Autores

A operação de transformação que melhora a nitidez de uma imagem é a adição desta com seu laplaciano. A implementação no DIP apresenta ao usuário uma máscara 3 x 3 com coeficientes 
similares aos da Figura 5, mas permite a digitação de outros valores. Portanto, a imagem de saída é obtida usando:

$$
g(x, y)=\left\{\begin{array}{l}
f(x, y)-\nabla^{2} f(x, y), \text { se } w_{00}<0 \\
f(x, y)+\nabla^{2} f(x, y), \text { se } w_{00}>0
\end{array},\right.
$$

Onde $w_{00}$ é o elemento central da máscara.

A Figura 6a foi obtida a partir da Figura 2a usando (6) com a abordagem RGB; a Figura $6 \mathrm{~b}$ foi obtida com a abordagem HSI. Novamente, a diferença entre os resultados RGB e HSI é notável.

Figura 6: Aumento da nitidez da Fig. 2a obtido com o laplaciano. (a) Abordagem RGB. (b) Abordagem HSI.

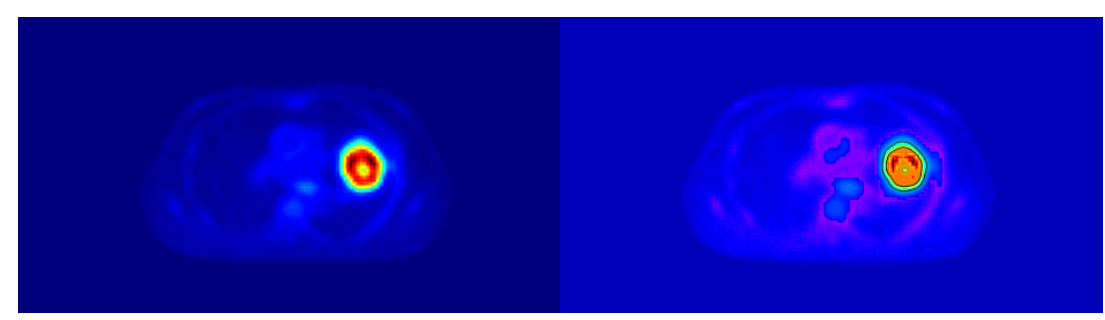

Fonte: Autores

\section{RESULTADOS E DISCUSSÕES}

Novamente a Fig. 2a foi usada para ilustrar o processo de segmentação na abordagem RGB. A Fig. 11a mostra a Fig. 2a onde, com o mouse, foi selecionado um retângulo contendo amostras de cores com predominância das componentes $\mathrm{G}$ e B (a cor ciano pura tem $\left[R\right.$ G B] $=\left[\begin{array}{ll}0 & 255\end{array}\right.$ 255]). A cor média, $\mathbf{a}=$ [32 211218 ], foi calculada tomando a média das componentes das cores no interior do retângulo mostrado na Fig. 11a. As Fig. 11b, c e d mostram outros dados necessários à implementação no software DIP usando a abordagem do paralelepípedo ilustrada na Fig. 10b. A Fig. 11 e mostra o resultado da segmentação. 
Figura 11: Segmentação na abordagem RGB. (a) Fig. 2 a com amostra das cores selecionada no retângulo. $(b, c, d)$ Janelas para obtenção e ajustes dos dados de entrada. (e) Imagem segmentada.
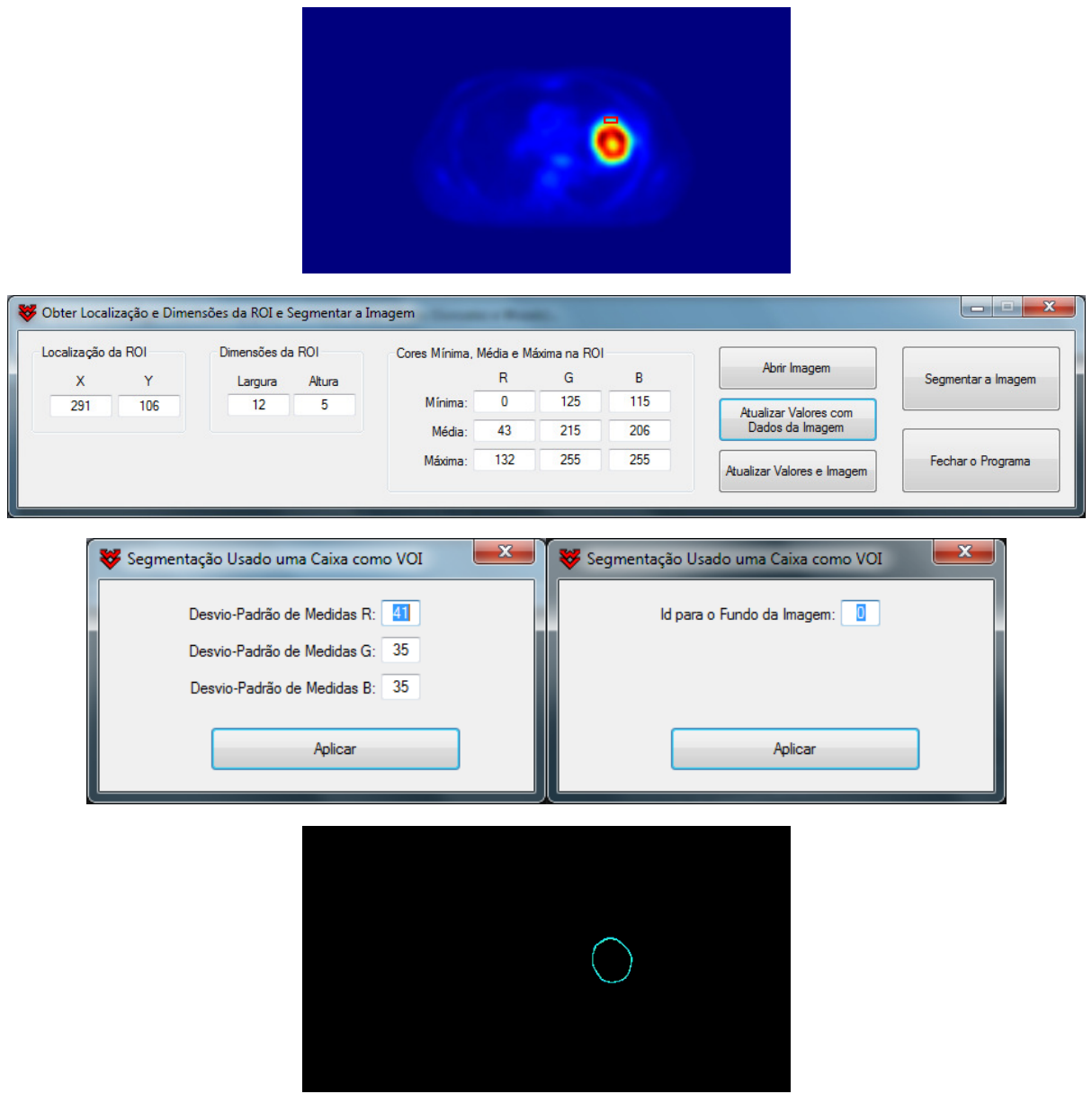

Fonte: Autores

A segmentação da região tumoral pode ser feita por etapas. A Fig. 12 mostra a separação entre o corpo e o fundo da Fig. 2a. Primeiramente, foi obtida a $2^{\mathrm{a}}$ imagem, usando a abordagem RGB com uma amostra de cores do fundo da $1^{a}$ imagem. Então foi obtida a imagem da diferença. A imagem obtida tem fundo uniforme, com $\mathrm{R}=\mathrm{G}=\mathrm{B}=0$. Isto evidencia que as imagens disponíveis não possuem ruídos ou artefatos indesejados. Consequentemente, não há necessidade de pré-processamentos antes de realizar a segmentação. A Fig. 13 mostra a segmentação do tumor na imagem de saída da Fig. 12. A Fig. 13a foi obtida tomando uma amostra da região do 
corpo sem o tumor e a Fig. 13b é a imagem da diferença entre a imagem de saída da Fig. 12 e a Fig. 13a.

Figura 12: Segmentação do corpo.

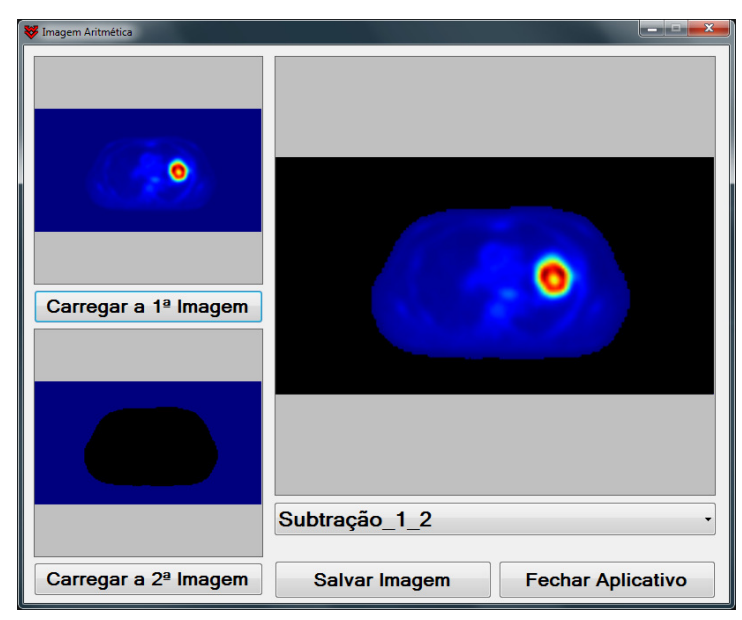

Fonte: Autores

Figura 13: Segmentação do tumor.

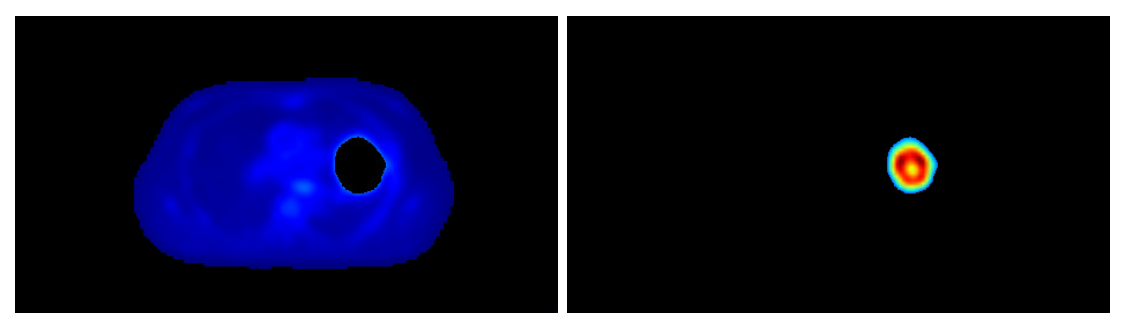

. Fonte: Autores

\section{CONCLUSÕES}

Como se vê nas Fig. 11e e 13b, a abordagem RGB é a mais indicada para segmentação de imagens PET. Imagens como estas podem ser associadas a valores de concentração de atividade e, como isso, é possível construir mapa de cores. Na sequência dos estudos do GDN serão implementadas as técnicas para segmentar o volume tumoral. As imagens segmentadas serão usadas para inserir tumores em fantomas de voxels já desenvolvidos e produzir os fantomas patológicos para avaliações dosimétricas. Sabendo-se a quantidade de voxels segmentados e o 
volume dos órgãos do fantoma "sadio", vai ser possível estimar o volume relativo do tumor. Tais imagens também serão úteis para quantificar concentrações de atividade não uniformes para otimizar simulações envolvendo dosimetria interna.

\section{AGRADECIMENTOS}

Os autores agradecem ao IFPE Campus Recife e CNPq pelo apoio financeiro.

\section{REFERÊNCIAS}

1. R. C. Gonzalez and R. E. WOODS, Digital Image Processing, 2nd ed., Prentice Hall, Upper Saddle River, New Jersey, USA (2002).

2. J. W. Vieira, and F. R. A. Lima, "A Software to Digital Image Processing to be Used in the Voxel Phantom Development", Cellular and Molecular Biology, 55, pp. 16-22 (2009). 